\title{
Automatic Material Estimation by Translating BIM Data into ERP Readable Data for Panelized Residential Construction
}

\author{
Meng Wang ${ }^{1}$, SangJun Ahn ${ }^{2}$, Youyi Zhang ${ }^{3}$, Mohammed Sadiq Altaf \\ ${ }^{4}$, Mohamed Al-hussein ${ }^{5}$, and Yongsheng $\mathrm{Ma}^{6 *}$ \\ ${ }^{1}$ M.Sc. Research Engineer, Department of Mechanical Engineering, University of Alberta \\ ${ }^{2}$ Ph.D. Student, Department of Civil \& Environmental Engineering, University of Alberta \\ ${ }^{3}$ M.Sc. Estimator, ACQBUILT - Landmark Homes, Edmonton \\ ${ }^{4}$ Post-Doc. fellow, Department of Civil \& Environmental Engineering, University of Alberta \\ ${ }^{5}$ Professor, Ph.D., Department of Civil \& Environmental Engineering, University of Alberta \\ ${ }^{6}$ Professor, Ph.D., Department of Mechanical Engineering, University of Alberta \\ "Corresponding author's e-mail: yongsheng.ma@ualberta.ca
}

\begin{abstract}
Offsite construction methods have been promoted and recognized globally as advanced construction techniques. Panelization, a two-dimensional form of offsite construction has brought about many benefits including lower transportation requirements and design flexibilities. In North America, the panelized construction technique has become popular particularly for wood-frame wall panels for residential construction. However, although utilizing this advanced construction method can greatly improve the working environment and productivity, the conventional mentality in construction, which overlooks the value of an automated management system to support offsite prefabrication and onsite installation, hinders its potential. An automatic material estimation system can capture all computer-aided design (CAD) data from building information modeling (BIM) files automatically and convert them into enterprise resource planning (ERP) readable data so that the ERP system can be updated for the purpose of material estimation. With the assistance of a feature-based modeling method, the proposed automatic material estimation system can save huge amounts of manual work for quantity take-off and all required CAD data can be captured by the system in a few seconds. Although highly dynamic market demands result in continual changes in design, production plan, schedule, and inventory levels, the adoption of an automatic material estimation system could account for these dynamic changes in order to maximize production. Therefore, in this paper, an automatic material estimation system is proposed in order to enhance the design and estimation process in the manufacturing environment. The proposed system is introduced and the development of supportive software is described. The proposed system is implemented as a case study in a panelized construction manufacturing facility, ACQBUILT, Inc., based in Edmonton, Alberta, Canada.
\end{abstract}

\section{KEYWORDS}

Panelized Construction; Building Information Modelling; Quantity Take-off; Feature-based Modelling

\section{INTRODUCTION}

The industrialization of prefabrication construction started a few years ago and has been continuously drawing attention from the construction industry due to its cost and schedule 
advantages over a traditional stick-built construction method (Babič et al., 2010). Pre-fabrication is a term used to describe the use of factory-produced pre-engineered building components such as two-dimensional components (panels) or when volumetric modules are delivered to the construction site for final assembly processes (Pasquire et al., 2002). Not only has prefabrication construction been deployed in the oil and gas industry, it has also been applied in residential building construction where each project can be panelized to reduce site cost and time while maintaining flexibility in designs. Panelization, a specific form of prefabrication construction, has emerged as an efficient and economical alternative approach to traditional stick-built construction (Liu et al., 2015), and it can achieve its efficiency by breaking down the residential projects into walls, floors and roof panels in manufacturing processes instead of on-site stick built. With panelization, as an approach, many potential benefits have been identified including reducing waste, increasing productivity, and creating a better controlled working environment, and many drawbacks of traditional stick-built building construction can be improved upon: for example, time consuming processes (e.g., onsite framing) can be eliminated; data accuracy (e.g., machine built) can be improved; and onsite labor costs can be reduced (Paevere et al., 2007).

However, although the advantages of panelized construction are numerous, project estimation processes for panelized construction have not yet been improved, and manual estimation is still being utilized - the same manual processes that are used in traditional stick-built construction. To improve the manual estimation processes, information technologies (IT) such as building information model (BIM) and enterprise resource planning (ERP) have been widely adopted in construction industries, but a proper integration between different IT systems has not been well studied before, and the typical estimation practice in the industry requires another manual process to incorporate these different IT systems.

Thus, to overcome the identified integration challenges between the BIM and ERP systems, this study proposes an automated material estimation system (hereinafter referred to as framework), which functions as a BIM data conversion framework, to seamlessly integrate ERP system in construction project estimation processes. To verify the framework, BIM data from actual panelized residential projects are tested to evaluate the overall process improvements in terms of errors and accuracy of the framework.

\section{RESEARCH BACKGROUND}

The BIM is an information technology that has been widely applied in the modern Architecture, Engineering and Construction (AEC) industry in terms of increasing productivity and enhancing communication. BIM contains enriched information required throughout the product lifecycle in terms of scheduling, analyses, cost, product etc., which have a significant role in project planning and analysis. It is object-oriented design and much more than just a database for the building model. Building structures are presented in panels as floors, walls (both exterior and interior), and roofs. Kim et al. (2013) proposed a prototype in order to automate construction scheduling, later Chen et al. (2013) developed a CAD-based system to improve the schedule accuracy and the CAD model provided quantity take-offs for process simulation purposes, but only a few studies have been dedicated to improving construction estimation processes using BIM data and enterprise resource planning (ERP) system (Abanda et al., 2017; Choi et al., 2015). Although the benefits of panelized construction have been playing a significant role in the off-site construction industry, the material's complexity and variability are the main factors that stop the design and manufacturing process from realizing the full potential benefits of this approach. To assist panelized construction companies to fully reach their potential so that BIM data from CAD 
systems can be automatically extracted and directly entered into the existing commercial ERP system, feature-based modelling as an integration mechanism has been proposed and a framework has been developed to automate this process instead of traditional manual work. Thus, by implementing this integrated system framework, manual work can be eliminated and issues including lack of accuracy and the associated high labor cost can be all improved. The following sections will discuss the proposed framework and the case study on the actual panelized residential projects.

\section{METHODOLOGY}

To achieve the automatic conversion of BIM data into ERP system, the framework in Figure 1 is proposed in this study.

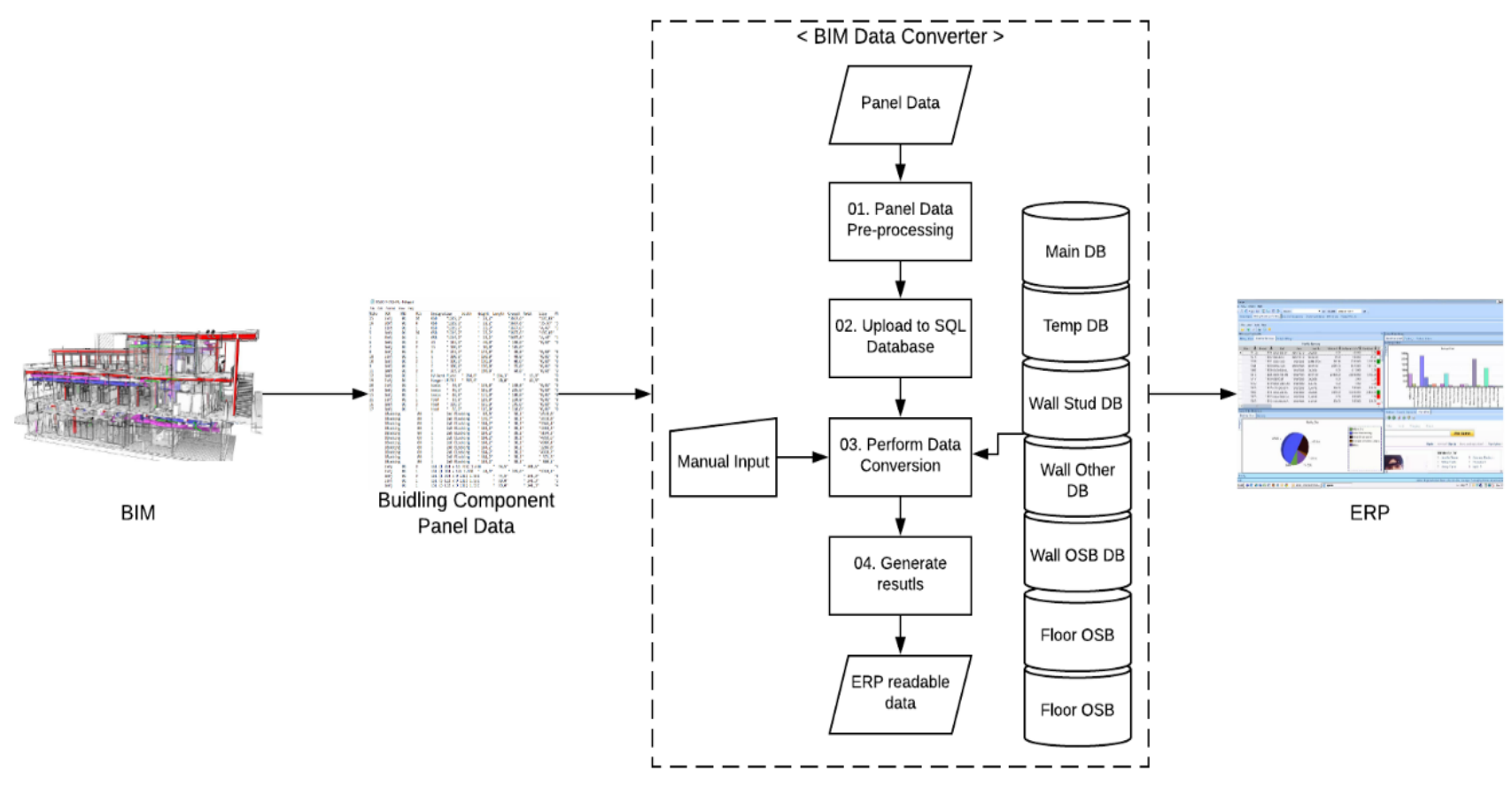

Figure 1. Overview of the proposed framework for converting BIM/CAD readable data into ERP system

Once a building modelling process is completed in BIM environment (e.g., Autodesk Revit), each building component information (e.g., materials) can be extracted from the model in different file formats. In this study, a text file format (.txt) is used as the file format of the building component information. Each building text file data contain 53 different columns regarding the materials that are used for building the model. For example, each material has an identification code, dimension, weight, volume, floor, material count, etc., and for the conversion purpose, this research utilizes 10 data columns to perform the task. More details on the selected data columns will be explained in the panel data pre-processing section. Next, the select building information is entered into the BIM data conversion module as shown in Figure 1. The module has four main processes that convert raw BIM data to the ERP readable format by assigning correct part numbers to each material. The part number is a unique identification code that is used in the ERP system for each material. The entered data in the module needs to be cleaned and reorganized before entering the 
following processes for the purpose of efficiency. The 10 different data columns from the raw BIM data are selected and the rest of columns are deleted from the data. After the cleaning processes, the cleaned data is uploaded to a local structured query language (SQL) database for the efficient conversion processes. The SQL database is known for its fast query processing in the case of large data (Cattell, 2011). The data conversion processes are a major step in the conversion module and a simple graphical user interface is developed to control and visualize conversion processes and outcomes, as shown in Figure 2. The conversion processes use seven database tables that support decision making processes for assigning correct job numbers to each material in the BIM file. Stud, floor, oriented strand board (OSB), and wall database tables are prepared tables and each table contains dimensional filtering information such as length, height, and width to distinguish different materials. After the filtering processes, part numbers are assigned to each material and grouped together for final outcomes.

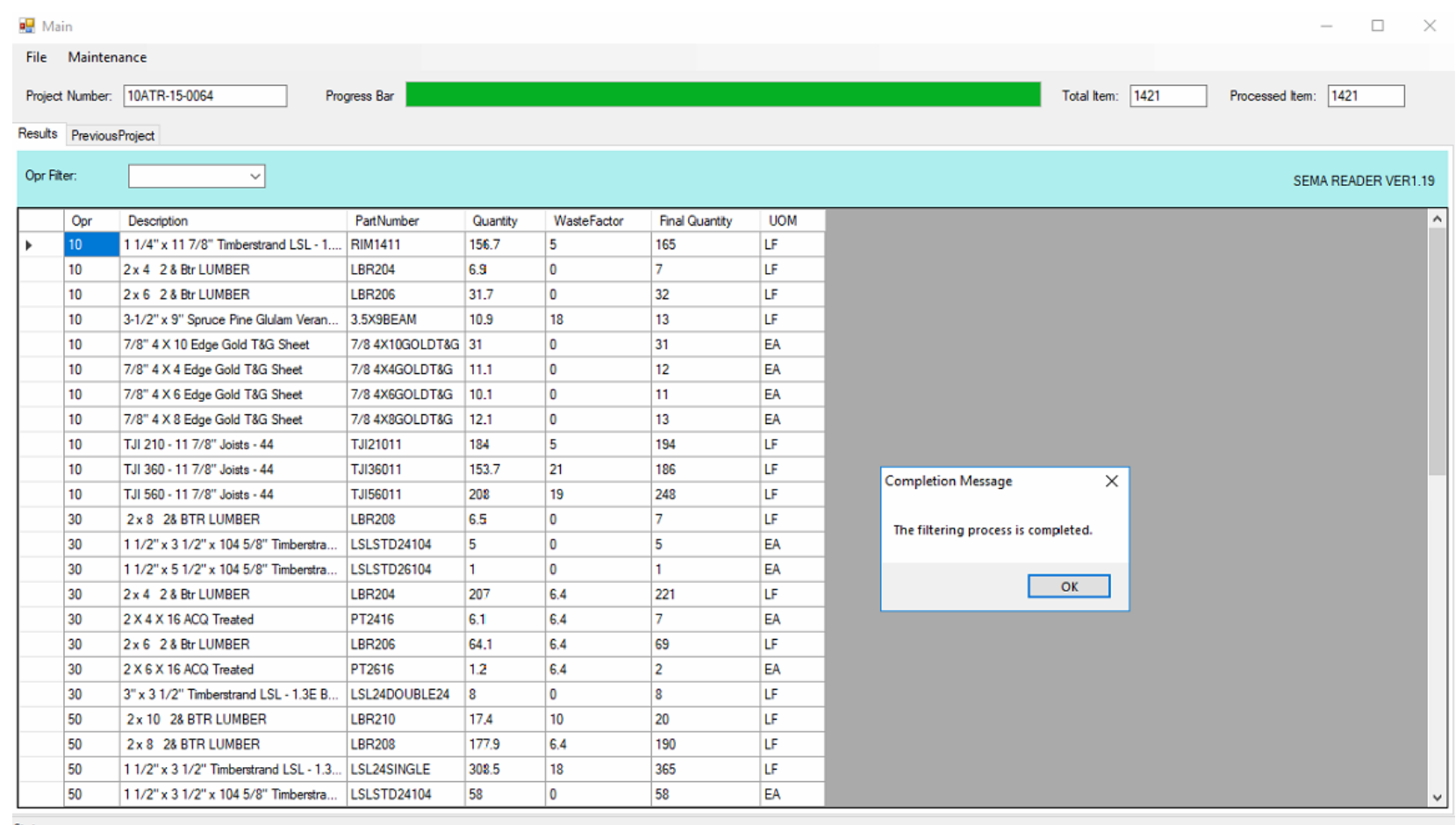

Figure 2. The GUI for the BIM data conversion processes

\section{The BIM data conversion module}

The conversion module is the core that allows the ERP system to read the BIM data. The module consists of four steps and the third step, which performs filtering and assigning of a part number to each material, is discussed in detail in this section. This step utilizes the developed algorithm, shown in Figure 3. The algorithm is written by using the C\# language in Microsoft Visual Studio 2017. 


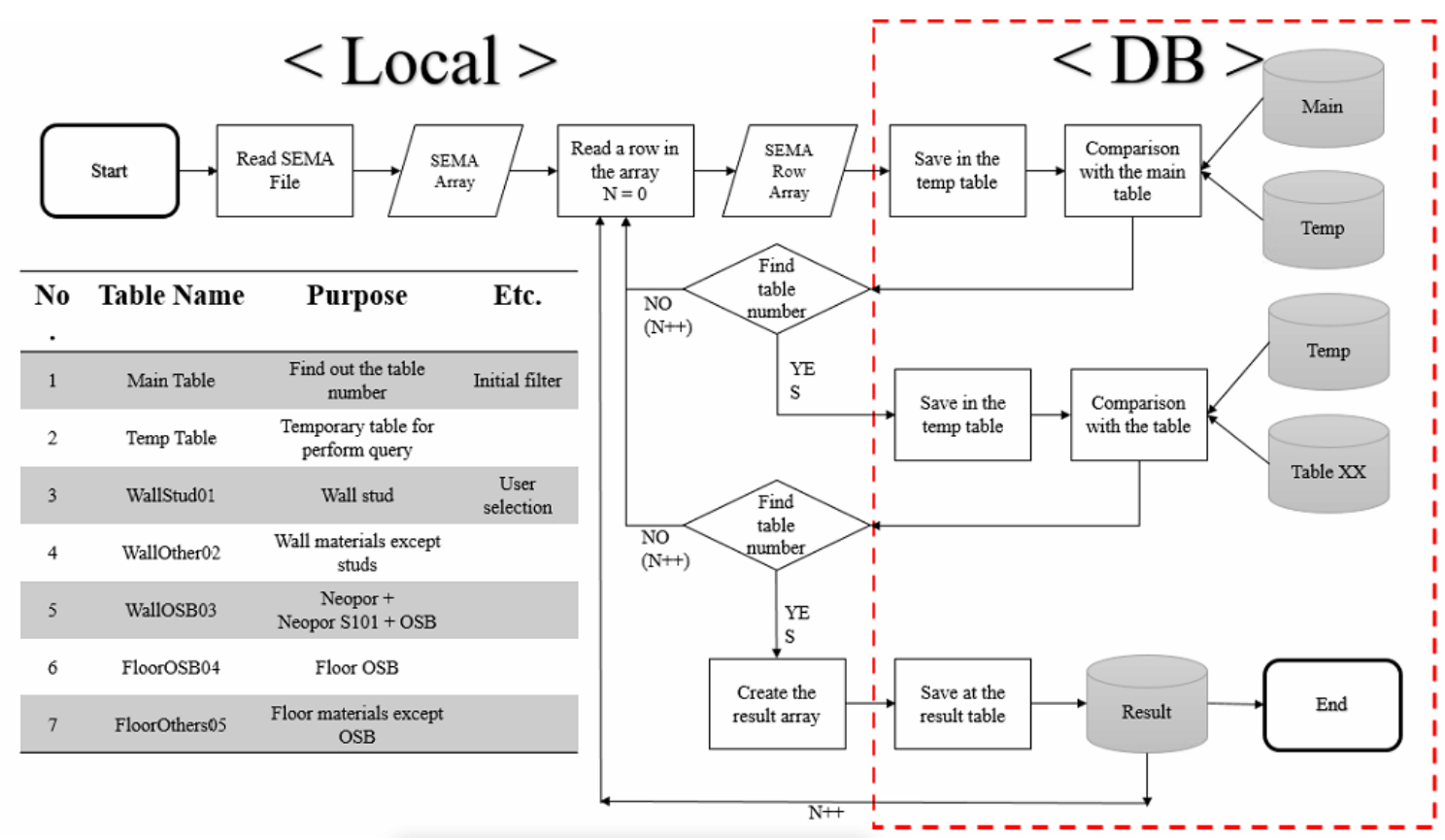

Figure 3. The developed BIM data conversion algorithm

Initial process is started by acquiring the BIM data in text file format, and the file is stored in a multi-dimensional array format in the algorithm. Once the array is checked for any special characters (e.g., parenthesis, quotation marks, apostrophe, and etc.) that could create errors during the algorithm processes by reading each string data, the first row of the array is selected for initial filtering processes that distinguish types of materials such as wall stud, wall others, wall OSB, floor OSB, and floor others, as shown in Figure 3. This process provides a next designation of the data in the row that requires further analysis to accurately assign the part number. Due to the usage of the new materials, the algorithm may not be able to provide a database table to a new material. In this case, the missing data algorithm is also developed to safely enter new data into suitable data tables while preventing data duplication or overwrite issues. After the correct data table is assigned to the data, the query is performed to find out a correct part number match for the materials and the results are stored in the result data table in the SQL database. Using a loop structure in the algorithm, these processes keep repeating until the BIM data row is null condition.

\section{Missing material in the data tables}

The situation where the algorithm cannot find a match for new construction materials can happen frequently for a new building project such as a custom house. This case is an inevitable situation because each building project cannot use the same materials, thus efficient and safe ways to enter new data into the data tables are important tasks to improve adaptability of the database. To detect and enter new data, the algorithm shown in Figure 4 is developed to accommodate these situations. 


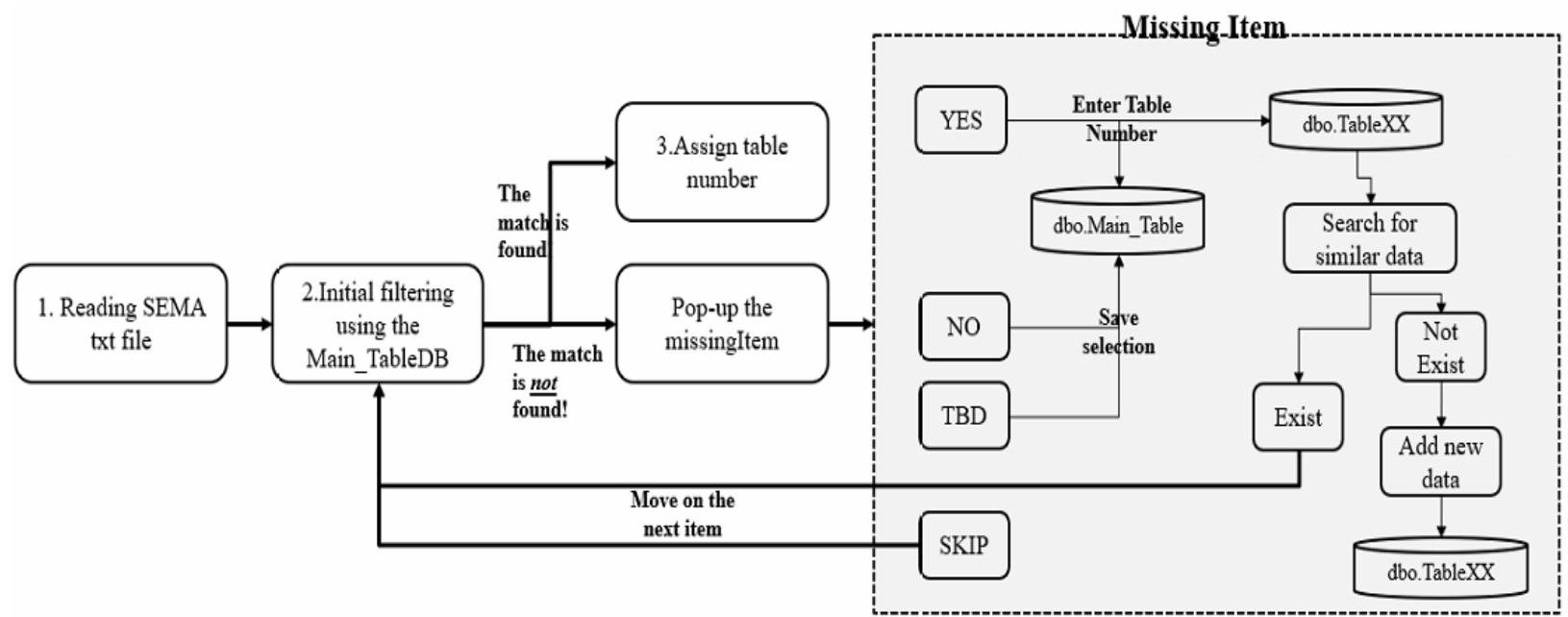

Figure 4. The missing data detection and entry algorithm

This missing data algorithm is processed when the main conversion algorithm in Figure 3 cannot find a match. Once the main algorithm cannot find the match, the missing data algorithm looks for similar items in different tables to show the user a potential similar match by comparing item's material name, description, floor level, and dimensions using multiple SQL queries. Sometimes, the same two items can have different names due to mistakes made in the initial development of the data tables. After the finding of similar matches and if a user still cannot find a match, then manual entry of the data is allowed and the main algorithm is repeated by moving back to the preview data row in the BIM data to provide a match.

\section{CASE STUDY: RESULTS AND DISCUSSION}

The proposed framework is applied on five different panelized home construction projects in order to compare its performance in terms of conversion accuracy against the ground truth values by counting in-correctly converted items. For example, total number of in-correct items is divided by total number of items to calculate percent error as in Table 1. The five projects are randomly selected among projects that have correct conversion values. The comparison results are presented in Table 1 and show that an average of 7.9\% conversion errors are estimated from the comparison. The lowest error is observed in the smallest house project, which is detached garage house type, while on the other hand, the largest error is found in the largest attached garage house project. This performance gap can be explained by a lack of data in a large house project. The amount of data for small projects is relatively higher than that for large homes (e.g., over 2,500 $\mathrm{ft}^{2}$ ); as a result, more comprehensive data for small homes are collected in the database.

Table 1. The error percentage (\%) between the proposed framework and the true values.

\begin{tabular}{cccccccc}
\hline OPR\# & Sq. Ft & Wall Stud & Wall Others & Wall OSB & Floor OSB & Floor Others & SUM \\
\hline Project\#1 & 1544 & 1.5 & 1 & 0.8 & 2 & 0.3 & 5.6 \\
Project\#2 & 2639 & 1 & 2 & 1.8 & 2.1 & 1.8 & 8.7 \\
Project\#3 & 1648 & 1.4 & 1 & 1.7 & 1.8 & 1.5 & 7.4 \\
Project\#4 & 3015 & 1.6 & 2 & 1.1 & 3.5 & 1.7 & 9.9 \\
Project\#5 & 2508 & 1.8 & 1 & 1.3 & 2.1 & 2.0 & 8.1 \\
\hline
\end{tabular}


Among the different building components shown in Figure 5, an average of 1.5\% conversion errors is observed from all areas. All areas have a similar error percentage except the floor OSB area. The floor OSB area has 2.3\% error and it was 53\% greater than the average error. It is suspected that the main reason for the high error in the floor OSB is due to the proposed framework not recognizing OSB materials in the side sections of the floor panels. Since the BIM model does not include the materials, the framework is unable to perform the conversion by reading the BIM data.

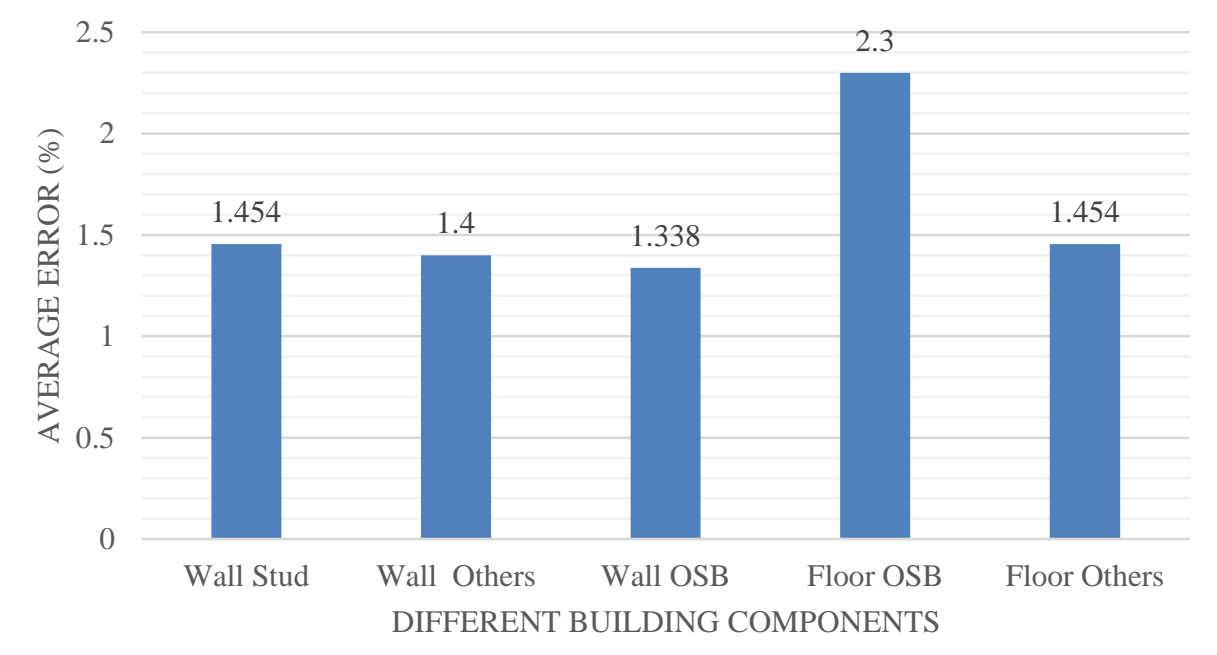

Figure 5. Average errors in percentage in different building component areas

Considering the overall conversion error of $7.9 \%$, this proposed framework could improve its performance by more BIM data being added into the database. More data in the database will make the framework more versatile in various future jobs. However, the required time to perform the conversions is significantly smaller than previous manual conversion processes. The framework takes less than 1 minute to perform the conversions of the large house project. Exact manual conversion times have not been measured due to differences in individual productivity, but it would be estimated around 20 to 30 minutes. Therefore, this framework can significantly reduce conversion times while connecting BIM and ERP system together. The current issue with the high conversion error can be easily improved by increasing the size of the database. Nowadays, more construction companies are adopting more information technologies to improve work efficiency and this framework will improve data transferring between different software platforms such as BIM and ERP.

\section{CONCLUSION}

This paper proposes a framework to accomplish BIM data conversion into ERP readable data format by assigning a part number for each construction material. The BIM data from a 3D model is extracted in text file format and the data is entered into the conversion module. The conversion module utilizes the SQL database to improve speed of conversion processes in case of a large BIM data size. The comparison results between the proposed framework and the true values from manual conversion processes are compared. Even though the conversion errors are relatively higher than the true values, the time saving offered by the framework can outweigh the issues with the errors, which can be reduced by enlarging the database. In the future, a machine learning 
technique can also be incorporated into the framework to further improve performance of the conversion. The central limitation of this research is the lack of test samples. More samples will provide more accurate evaluation of the performance of the framework. The selection of the project samples was limited by availability of correctly converted data.

\section{ACKNOWLEDGEMENTS}

We gratefully acknowledge the financial support of the Natural Sciences and Engineering Research Council of Canada (NSERC).

\section{REFERENCE}

Abanda, F. H., Kamsu-Foguem, B., \& Tah, J. H. M. (2017). BIM-new rules of measurement ontology for construction cost estimation. Engineering Science and Technology, an International Journal, 20(2), 443-459.

Babič, N. Č, Podbreznik, P., \& Rebolj, D. (2010). "Integrating resource production and construction using BIM." Automation in Construction, 19(5), 539-543.

Choi, J., Kim, H., \& Kim, I. (2015). Open BIM-based quantity take-off system for schematic estimation of building frame in early design stage. Journal of Computational Design and Engineering, 2(1), 16-25.

Pasquire, C.L., and Gibb, A.G.F. (2002). "Considerations for assessing the benefits of standardization and pre-assembly in construction.” Journal of Financial Management of Property and Construction, 7(3), 151-161.

Liu, H., Altaf, M. S., Lei, Z., Lu, M., and Al-Hussein, M. (2015). "Automated production planning in panelized construction enabled by integrating discrete-event simulation and BIM." Proceedings, International Construction Specialty Conference, 048, 1-10.

Paevere, P. and MacKenzie, C. (2007). Emerging Technologies and Timber Products in Construction: Compendium of Products and Technologies. Technical report commissioned by the Australian Forest and Wood Products Research and Development Corporation. 244 pages.

Kim, H., Anderson, K., Lee, S., and Hildreth, J. (2013). "Generating construction schedules through automatic data extraction using open BIM (building information modeling) technology." Automation in Construction, 35, 285-295.

Chen, S.M., Griffis, F.H., and Chen, P.H., and Chang, L.M. (2013). "A framework for an automated and integrated project scheduling and management system." Automation in Construction, 35, 89-110.

Cattell, R. (2011). "Scalable SQL and NoSQL data stores." Acm Sigmod Record, 39(4), 12-27. 